BMJ Open Sport \& Exercise Medicine

\title{
Quantifying exposure to running for meaningful insights into running- related injuries
}

\author{
John J Davis IV, Allison H Gruber
}

To cite: Davis IV JJ, Gruber AH. Quantifying exposure to running for meaningful insights into running-related injuries. BMJ Open Sport \& Exercise Medicine 2019;5:e000613. doi:10.1136/ bmjsem-2019-000613

Accepted
Check for updates

C Author(s) (or their employer(s)) 2019. Re-use permitted under CC BY-NC. No commercial re-use. See rights and permissions. Published by BMJ.

Department of Kinesiology, School of Public Health, Indiana University, Bloomington, Indiana, USA

Correspondence to Dr Allison H Gruber; ahgruber@indiana.edu

\section{ABSTRACT}

The very term 'running-related overuse injury' implies the importance of 'use', or exposure, to running. Risk factors for running-related injury can be better understood when exposure to running is quantified using either external or internal training loads. The advent of objective methods for quantifying exposure to running, such as global positioning system watches, smartphones, commercial activity monitors and research-grade wearable sensors, make it possible for researchers, coaches and clinicians to track exposure to running with unprecedented detail. This viewpoint discusses practical issues surrounding the use and analysis of data from such devices, including how wearable devices can be used to assess both internal and external training loads. We advocate for an integrative approach where data from multiple sources are used in combination to directly measure exposure to running in diverse settings.

Research into running-related overuse injury is based on a simple premise: overuse injury occurs when biological tissue experiences repeated loading exceeding its structural capacity. ${ }^{12}$ Given this assumption, risk factors for running-related injury are best examined as a function of running 'exposure', using measurements of the internal or external load experienced by the body. There is growing evidence that exposure is of paramount importance for understanding overuse injury in sport and for planning training or rehabilitation programmes for individual athletes (for examples, see Refs. 3-5).

\section{WHY MEASURE EXPOSURE?}

Imagine a hypothetical prospective study of healthy runners in which Participant A runs $20 \mathrm{~km}$-per-week and suffers an injury during the first week of follow-up, while Participant B runs $20 \mathrm{~km}$-per-week and suffers an injury during the 52nd week of follow-up. Categorising both runners as injured during follow-up' implies that both had an equal risk for injury. However, we might suspect that Participant A had a greater risk of injury because he or she completed a smaller total training load prior to the emergence of injury

\section{Key points}

- Running-related overuse injury should be examined as a function of 'exposure' to running.

- Exposure can be quantified using metrics of external training load (such as distance, duration or steps taken while running) or metrics of internal training load (such as rated perceived exertion or heart rate).

- A range of wearable devices can be used to directly and objectively measure running exposure, which can better reflect the actual or true training performed than self-report training diaries and surveys.

- Integrating exposure data from multiple sources can generate rich, informative datasets and lead to better insights into running-related injuries.

than Participant B. In this example, external training load, as measured by kilometres-per-week, provides valuable information on running exposure.

However, if Participant A trains at a faster pace than Participant B, the effective exposures to running during each week might no longer be equivalent-fast running may incur more cumulative tissue damage per kilometre. $^{6} 7$ Moreover, internal training load can vary even when external training load is constant. Suppose Participants C and $\mathrm{D}$ both run $20 \mathrm{~km}$-per-week at a pace of 6 min-per-kilometre. If this pace elicits $70 \%$ of maximum heart rate in Participant $\mathrm{C}$, but $85 \%$ in Participant D, these runners are accumulating different internal training loads, despite having equal external training loads. Internal training load contributes to fitness and performance, which may be associated with injury risk. ${ }^{89}$ Thus, both external and internal training loads should be considered when studying injury risk or when planning a training or rehabilitation programme.

\section{QUANTIFYING EXPOSURE WITH INTERNAL TRAINING LOAD}

Internal training load metrics capture information about the runner's physiological effort during training and can help assess 
overload. Traditional metrics of physiological effort like submaximal oxygen consumption or blood lactate are impractical for monitoring training load repeatedly in the field; rated perceived exertion (RPE) and heart rate are more practical tools for assessing internal training load, while still retaining the reliability needed for research. ${ }^{1011}$

\section{QUANTIFYING EXPOSURE WITH EXTERNAL TRAINING LOAD}

External metrics for training load include time spent, distance covered or steps taken while running. Distance and duration are easy to measure and understand, but from a biomechanical perspective, steps taken while running may be a better estimate of the volume of mechanical loading faced by the body. ${ }^{1}$ Running speed is the primary method of quantifying the intensity of an external load, although the caveats discussed above regarding the relationship between pace and internal load.

\section{HOW SHOULD THE RESEARCHER, COACH OR CLINICIAN MEASURE EXPOSURE TO RUNNING?}

The advantages of exposure-based research on running injuries include the ability to quantify 'training errors' and the ability to identify variables that modify the relationship between training load and injury risk. Many references exist for guidance on how to analyse data under an exposure-based framework..$^{3-5}$ The remainder of this article discusses practical considerations surrounding methods for measuring running exposure and an integrative approach that combines multiple data sources for better insights into injury risk.

\section{TRAINING DIARIES}

Training diaries, including surveys, are the most obvious way to assess external training load, but self-report data compares poorly to the wide range of objective methods for measuring external training load. Results from one study of 100 recreational runners suggest that self-reported distance covered in a single training session can differ by $-28 \%$ to $+40 \%$ of the true distance. ${ }^{12}$ Because self-reported training volume can vary so drastically from the true value, drawing strong conclusions from self-report data alone is challenging. However, self-report training diaries are the best way to regularly assess RPE to quantify an athlete's internal training load and provide a convenient avenue for assessing other variables of interest, such as mood state, illness and injury. ${ }^{13} \mathrm{An}$ online, email, text message or app-based training diary can streamline the integration of self-report data with the sensor-based exposure metrics discussed below.

\section{GLOBAL POSITIONING SYSTEM (GPS) ENABLED DEVICES}

GPS-equipped smartphones and watches are used to objectively measure running time and distance covered in prospective running research. Distance, time and speed provided by GPS devices can be easily interpreted by researchers, clinicians and coaches without special software or training. GPS is most accurate on straight paths with a clear view of the sky, while accuracy deteriorates in urban or heavily forested areas. Even in suboptimal environments, GPS estimates distance covered with $\leq 6.2 \%$ error. ${ }^{14}$ GPS watches face two primary limitations: they cannot be used indoors, and users must manually activate the watch for each training session, which imposes a moderate participant burden.

\section{COMMERCIAL ACTIVITY MONITORS}

Commercially available activity monitors worn at the wrist (eg, Fitbit) or mounted on a runner's shoe (eg, RunScribe) combine automated data recording with smartphone-based data synchronisation, removing the need for manual recording of training sessions. These devices are accelerometer-based, so they work equally well indoors and outdoors. However, they rely on proprietary algorithms for estimating metrics like run duration, distance covered and average speed, which often have not been validated against gold standard measurements (eg, geodetic GPS equipment ${ }^{14}$ ). In situations where minimum burden on participants and maximum flexibility in training environments are desirable, a commercial activity monitor may be a practical choice for objective quantification of external training load.

Some commercial activity monitors can objectively quantify internal training load using heart rate, as measured by an optical sensor at the wrist or an electrical sensor on a chest strap. While chest-worn electrical sensors are considered the gold standard for heart rate, wrist-worn optical sensors only err by $3.3 \%-6.2 \%$ during running. ${ }^{15}$ Commercial activity monitors and researchgrade wearable sensors may offer the ability to collect additional data, including measures of sleep and non-running physical activity behaviour, which are important to monitor training recovery.

\section{RESEARCH-GRADE WEARABLE SENSORS}

Research-grade wearable sensors (eg, ActiGraph, Axivity, IMeasureU) are explicitly designed to capture parameters of interest to physical activity researchers and human movement scientists. Many research-grade devices provide raw, high-resolution data captured by on-board accelerometers and possibly gyroscopes and magnetometers as well.

While research-grade sensors capture richly detailed biomechanical information, working with the enormous amount of data generated is challenging. For basic exposure metrics, such as time spent or steps taken while running, an analyst must apply sophisticated step identification and activity recognition algorithms. However, access to raw data can enable the estimation of important biomechanical parameters that may modify the relationship between training load and injury, such as impact shock or ground reaction forces. ${ }^{16-18}$ Some commercial devices also measure biomechanical parameters, but do so using proprietary algorithms that may not be validated against gold-standard laboratory data. 


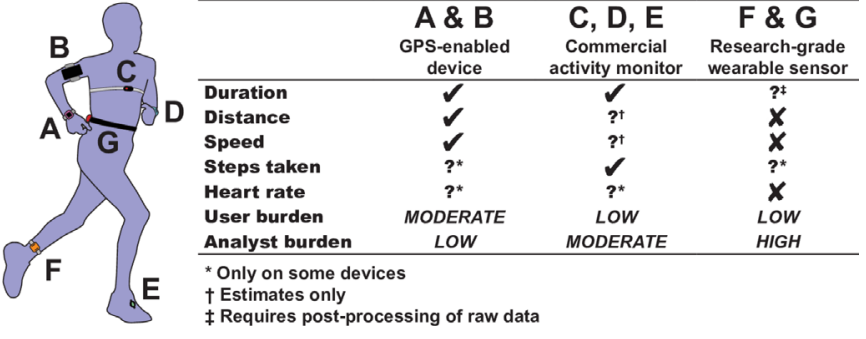

Figure 1 A runner's 'exposure' to running can be easily monitored through a variety of objective tools, including global positioning system (GPS) watches (A) and smartphones (B); commercial activity monitors worn at the chest (C), wrist (D) or shoe (E) and research-grade wearable sensors worn on the ankle $(\mathrm{F})$ or at the waist $(\mathrm{G})$.

While research-grade devices are an attractive option for data-driven investigations into mechanisms of running-related injury, the lack of automatically generated exposure metrics means these devices often do not meet the needs of clinicians and coaches, who require rapid data processing and output. Moreover, researchgrade wearable sensors can be prohibitively expensive.

\section{COMBINING DATA SOURCES}

Using multiple methods to measure different metrics of running exposure simultaneously can capture informative data at several levels of resolution (figure 1). For example, a prospective study might incorporate GPS watches, on-shoe commercial activity monitors, a waistworn research-grade accelerometer and a daily survey. This multilayered approach helps to defend against user error, reduces uncertainties associated with missing data and can sidestep difficulties associated with identifying and extracting periods of running from raw accelerometer-based sensor data. ${ }^{19} 20$ Synchronising data across multiple devices can be challenging, particularly in devices that measure raw sensor output (described elsewhere $^{21}$ ). Researchers, coaches and clinicians should weigh the benefits of collecting more data against the increases in data processing workload and athlete burden when considering how many devices to employ.

\section{TOWARDS STRONGER RESEARCH ON UNDERSTANDING RUNNING-RELATED INJURY}

Exposure metrics provide a useful framework for studying risk factors for running-related overuse injury. However, running exposure is one of many considerations within the complex causal pathways that lead to overuse injury. Variables such as footwear, sleep, nutrition, environment and training polarisation can be understood more clearly when framed as variables that modify the relationship between running exposure and risk of running-related injury. Gait must also be considered because it modulates the interaction of structure-specific load and external exposure to running. ${ }^{3}$ Unmodifiable factors (eg, age, sex, genetics, anatomy) must also be considered.

Prospective research, training programmes and rehabilitation protocols require precise estimates of running exposure. Fortunately, researchers, clinicians and coaches have a wide range of options to capture objective running exposure metrics. The relative advantages of training diaries, GPS-enabled devices, commercial activity monitors and research-grade wearable sensors are best used in combination to generate rich, informative and robust datasets.

Contributors Both authors made substantial contributions to the conception, drafting and revisions of this work.

Funding The authors did not receive a specific grant for this research from any funding agency in the public, commercial or not-for-profit sectors.

Competing interests None declared.

Patient consent for publication Not required.

Provenance and peer review Not commissioned; externally peer reviewed.

Open access This is an open access article distributed in accordance with the Creative Commons Attribution Non Commercial (CC BY-NC 4.0) license, which permits others to distribute, remix, adapt, build upon this work non-commercially, and license their derivative works on different terms, provided the original work is properly cited, appropriate credit is given, any changes made indicated, and the use is non-commercial. See: http://creativecommons.org/licenses/by-nc/4.0/.

ORCID iD

Allison H Gruber http://orcid.org/0000-0002-0750-5656

\section{REFERENCES}

1 Edwards WB. Modeling overuse injuries in sport as a mechanical fatigue phenomenon. Exerc Sport Sci Rev 2018;46:224-31.

2 Nielsen RO, Bertelsen ML, Møller M, et al. Training load and structure-specific load: applications for sport injury causality and data analyses. Br J Sports Med 2018;52:1016-7.

3 Nielsen Rasmus Østergaard, Malisoux L, Møller M, et al. Shedding light on the etiology of sports injuries: a look behind the scenes of time-to-event analyses. J Orthop Sports Phys Ther 2016;46:300-11.

4 Bahr R, Holme I. Risk factors for sports injuries -- a methodological approach. Br J Sports Med 2003;37:384-92.

5 Ullah S, Gabbett TJ, Finch CF. Statistical modelling for recurrent events: an application to sports injuries. Br J Sports Med 2014;48:1287-93.

6 Baggaley M, Brent Edwards W, Edwards WB. Effect of running speed on Achilles tendon injury potential: use of a weighted impulse measure. Denver, Colorado: American College of Sports Medicine Annual Meeting, 2017: 139.

7 Brent Edwards W, Taylor D, Rudolphi TJ, et al. Effects of running speed on a probabilistic stress fracture model. Clin Biomech 2010;25:372-7.

8 Manzi V, Iellamo F, Impellizzeri F, et al. Relation between individualized training impulses and performance in distance runners. Med Sci Sports Exerc 2009;41:2090-6.

9 Lisman PJ, Sarah J, Gribbin TC, et al. A systematic review of the association between physical fitness and musculoskeletal injury risk: part 1-cardiorespiratory endurance. J Strength Cond Res 2017;31:1744-57.

10 Haddad M, Stylianides G, Djaoui L, et al. Session-RPE method for training load monitoring: validity, ecological usefulness, and influencing factors. Front Neurosci 2017;11:612.

11 Foster C, Florhaug JA, Franklin J, et al. A new approach to monitoring exercise training. J Strength Cond Res 2001;15:109-15.

12 Dideriksen M, Soegaard C, Nielsen RO. Validity of self-reported running distance. J Strength Cond Res 2016;30:1592-6.

13 Clarsen B, Myklebust G, Bahr R. Development and validation of a new method for the registration of overuse injuries in sports injury epidemiology: the Oslo sports trauma research centre (OSTRC) overuse injury questionnaire. Br J Sports Med 2013;47:495-502.

14 Nielsen RO, Cederholm P, Buist I, et al. Can GPs be used to detect deleterious progression in training volume among runners? $J$ Strength Cond Res 2013;27:1471-8.

15 Stahl SE, An H-S, Dinkel DM, et al. How accurate are the wristbased heart rate monitors during walking and running activities? are they accurate enough? BMJ Open Sport Exerc Med 2016;2:e000106. 
16 Bergamini E, Picerno P, Pillet $\mathrm{H}$, et al. Estimation of temporal parameters during sprint running using a trunk-mounted inertial measurement unit. J Biomech 2012;45:1123-6.

17 Kiernan D, Hawkins DA, Manoukian MAC, et al. Accelerometerbased prediction of running injury in national collegiate athletic association track athletes. J Biomech 2018;73:201-9.

18 Neugebauer JM, Collins KH, Hawkins DA. Ground reaction force estimates from Actigraph GT3X+ hip accelerations. PLoS One 2014;9:e99023.
19 Van Buuren S. Flexible imputation of missing data. Chapman and Hall/CRC, 2018.

20 Benson LC, Ahamed NU, Kobsar D, et al. New considerations for collecting biomechanical data using wearable sensors: number of level runs to define a stable running pattern with a single IMU. $J$ Biomech 2019.

21 Karas M, Bai J, Strączkiewicz M, et al. Accelerometry data in health research: challenges and opportunities. Stat Biosci 2019:1-28. 